\title{
Discovery of proangiogenic CD44+mesenchymal cancer stem cells in an acute myeloid leukemia patient's bone marrow
}

\author{
Huynh Cao ${ }^{1 \dagger}$, Jeffrey Xiao ${ }^{2 \dagger}$, Mark E. Reeves ${ }^{1}$, Kimberly Payne ${ }^{3}$, Chien Shing Chen ${ }^{1}$, David J. Baylink²,
} Guido Marcucci ${ }^{4}$ and $Y i X^{1,2^{*}}$ (D)

\begin{abstract}
Here, we report a unique acute myeloid leukemia (AML) bone marrow-derived mesenchymal stem cell (MSC) with both mesenchymal and endothelial potential, which we have named Mesenchymal Cancer Stem Cells (MCSCs). These MCSCs are CD90-CD13-CD44+ and differ from MSCs in isolation, expansion, differentiation, immunophenotype, and cytokine release profile. Furthermore, blocking CD44 inhibited the proliferation and cluster formation of early MCSCs with lower ICAM-1 protein levels. Similar CD90-CD44+ cancer stem cells have been reported in both gastric and breast cancers, which grew in floating spheres in vitro and exhibited mesenchymal features and high metastatic/tumorigenic capabilities in vivo. Our novel discovery provides the first evidence that certain AMLs may be comprised of both hematopoietic and stromal malignant cells. Targeting MCSCs and their cytokine release has potential as a novel therapeutic approach in AML.
\end{abstract}

Keywords: Acute myeloid leukemia, Mesenchymal stem cells, Mesenchymal cancer stem cells, CD44, Angiogenesis, Microenvironment

\section{To the Editor}

Acute myeloid leukemia (AML) most commonly occurs in older adults [1] and has the lowest survival rate among all types of leukemia [2]. Mechanistically, AML is a hematopoietic cancer that involves a complex interplay among different types of bone marrow (BM) cells, cytokines, growth factors, and neural modulation-all of which contribute to the survival and growth of malignant Hematopoietic Stem Cells (HSCs) [3, 4]. Currently, most AML therapies have been developed to target malignant

\footnotetext{
* Correspondence: dyxu@llu.edu

${ }^{\dagger}$ Huynh Cao and Jeffrey Xiao are co-first authors.

'Division of Hematology and Oncology, Loma Linda University Medical Center, Loma Linda, CA, USA

${ }^{2}$ Regenerative Medicine, Department of Medicine, Loma Linda University, Loma Linda, CA, USA

Full list of author information is available at the end of the article
}

HSCs because of their significant role in initiating uncontrolled clonal proliferation and transforming the naïve BM niche to allow disease progression and treatment resistance [5]. However, the overall survival of relapsed or refractory AML patients has remained dismal for the past three decades [6]. Therefore, more research on the mechanisms of AML etiology and relapse are urgently needed to further the development of effective treatments.

A new concept of niche-induced oncogenesis has been proposed that is based on the observation that artificial genetic perturbation of mesenchymal stem cells (MSCs), and their progenies leads to leukemogenesis of myelodysplasia and AML in transgenic mice $[7,8]$. In this study from an AML patient BM, for the first time, we discovered previously unrecognized CD90-CD13-CD44+

(c) The Author(s). 2020 Open Access This article is licensed under a Creative Commons Attribution 4.0 International License, which permits use, sharing, adaptation, distribution and reproduction in any medium or format, as long as you give appropriate credit to the original author(s) and the source, provide a link to the Creative Commons licence, and indicate if changes were made. The images or other third party material in this article are included in the article's Creative Commons licence, unless indicated otherwise in a credit line to the material. If material is not included in the article's Creative Commons licence and your intended use is not permitted by statutory regulation or exceeds the permitted use, you will need to obtain permission directly from the copyright holder. To view a copy of this licence, visit http://creativecommons.org/licenses/by/4.0/. The Creative Commons Public Domain Dedication waiver (http://creativecommons.org/publicdomain/zero/1.0/) applies to the data made available in this article, unless otherwise stated in a credit line to the data. 
MSCs with tumorigenic proliferation and proangiogenic properties ex vivo.

The AML bone marrow donor is a 32-year-old female with a past medical history of diabetes and hypothyroidism. She was admitted to the emergency department with intermittent chest pain, shortness of breath, and gum bleeding, and diagnosed with AML with myelomonocytic differentiation. Peripheral blood smears showed leukocytosis with $52 \%$ blasts/equivalents and marked thrombocytopenia. FISH showed normal cytogenetics. An AML panel was positive for mutations of DNMT3A, NPM1, and NRAS without FLT3-ITD.

During ex vivo isolation of MSCs from this patient $\mathrm{BM}$, we observed two different cell populations. The early plastic-attached cells were CD90+CD13+CD44+ MSCs (Fig. 1(a1, a3, d1 upper panels)). The late attached cells in the non-adherent floating cells of MSC cultures became adherent after 7 or more days (Fig. 1(a2)). We named them mesenchymal cancer stem cells (MCSCs) because of their capabilities of

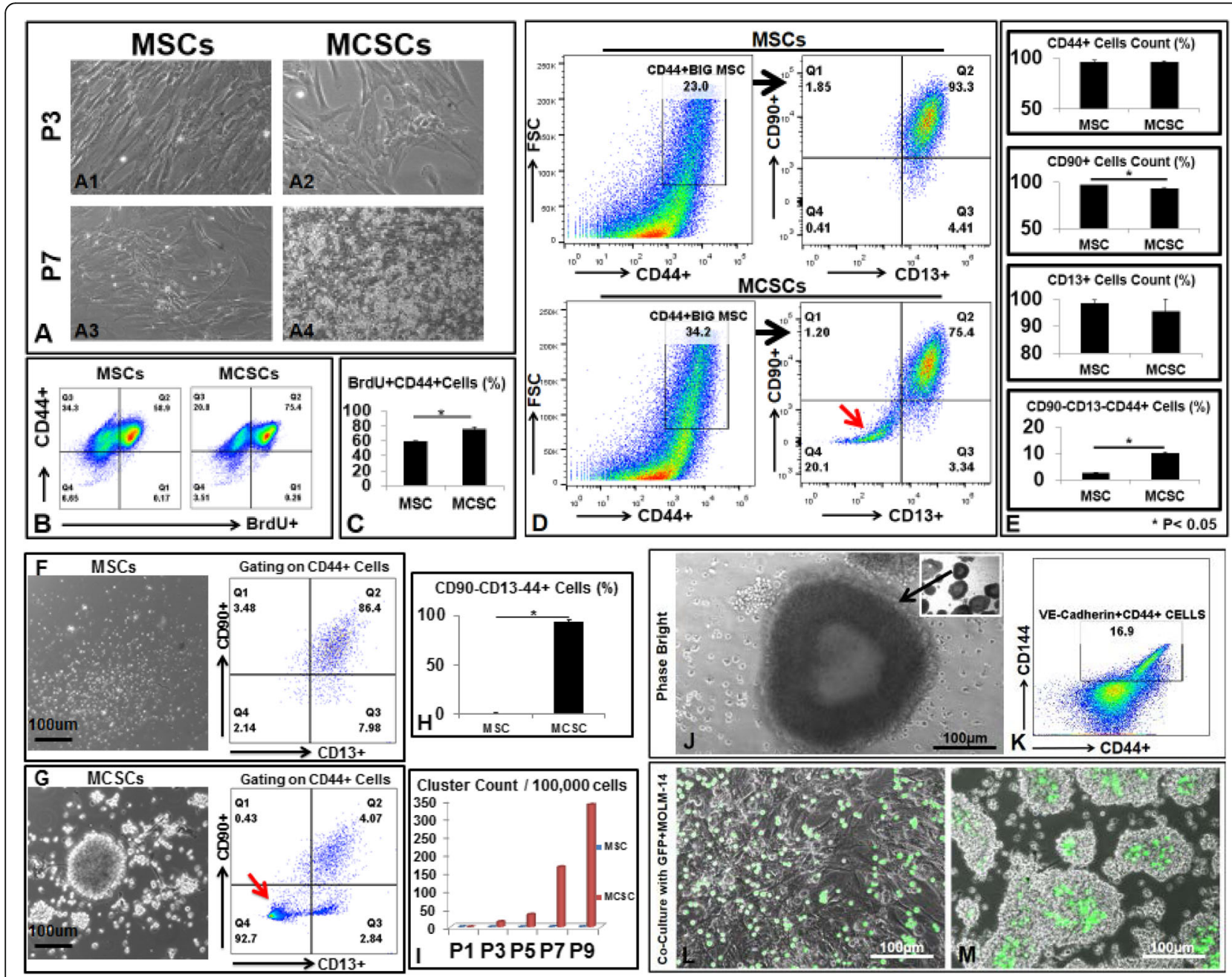

Fig. 1 Isolation and expansion of CD90-CD13-CD44+MCSCS from an AML patient bone marrow ex vivo. a Phase bright images of MSCS and MCSCs at different passages (P). b $24 \mathrm{~h}$ after culturing cells with BrdU, P7 MSCs, and P7 MCSCs were collected and analyzed by FACS for incorporation of BrdU. $\mathbf{c}$ Aggregate data showing percentage of BrdU+CD44+ cells in MSCs and MCSCs. d P3 MSCs and P3 MCSCs were analyzed by FACS for immunophenotype. Thick black arrows indicate gating strategy. The red arrow indicates the population of CD90-CD13-CD44+ cells. e Aggregate FACS data showing the percentage of MSCs and MCSCs positive for each biomarker. $\mathbf{f}$ Left: Phase bright image of floating cells from P5 MSC culture. Right: P5 MSCs were collected and analyzed by FACS. $\mathbf{g}$ Left: Phase bright image of floating cells from P5 MCSC culture. Right: P5 MCSCs were collected and analyzed by FACS. The red arrow indicates CD90-CD13-CD44+ cells. $\mathbf{h}$ Aggregate FACS data showing the percentage of CD90-CD13-CD44+ cells comprising the floating cell populations from MSCS and MCSCs. $\mathbf{i}$ Cumulative counts of clusters generated from MSC and MCSC cultures over time. $\mathbf{j}$ Inset: phase bright images of floating tube-like structure from P8 MCSC culture. Black thick arrows indicate the same round tube at $\times 2$ and $\times 10$ magnification. k P8 Floating MCSCS were analyzed by FACS for endothelial cell markers such as VE-Cadherin (CD144). I Phase bright image of GFP+MOLM-14 cells cultured with MSCs after 24h plating. $\mathbf{m}$ Phase bright image of GFP+MOLM-14 cells co-cultured with MCSCs after 24-h plating. Where applicable, data are means \pm SEM from each group and were analyzed by Student $t$ test. ${ }^{*} p<0.05 ; \mathrm{N}=3$. Scale bar $100 \mu \mathrm{m}$ 
multiple lineage differentiation (Supplementary Figure 1), rapid cell proliferation and self-renewal (Fig. 1b, c, Supplementary Figure 2), and release of high amounts of cytokines and growth factors reported to be essential for angiogenesis and blasts' growth. At early passages (P), there were about 20\% CD90-CD13-CD44+ cells within large-sized strong CD44+ MCSCs (Fig. 1d lower panels). MCSCs grew in plastic attachment patterns; however, the number of viable floating cells increased with passage (Fig. 1(A4)). After P7, most MCSCs were CD90-CD13-CD44+, expanded in floating cluster conditions (Fig. 1g, i), and generated tubelike vessel structures (Fig. $1 \mathrm{j}$ ) by expressing endothelial cell biomarkers like VE-Cadherin/CD144 (Fig. 1k). To understand the interaction between MCSCs and AML blasts, we plated MCSCs or MSCs with GFP-MOLM14. (AML blasts) together. After $24 \mathrm{~h}$ of $1: 1$ cocultures, GFP-MOLM-14 were found in the middle of floating clusters and surrounded by MCSCs (Fig. $1 \mathrm{~m}$ ), in contrast to their position on top of MSCs when cultured with MSCs only (Fig. 11). These data are consistent with a previous report that AML blasts integrate into vascular niches to progress and metastasize [9].

Finally, we performed a proteome assay of supernatants from MSC and MCSC cultures. The release of key angiogenic cytokines like VEGF and growth factors such as FGF basic and PDGF were significantly increased in the P7 MCSC culture (Fig. 2b). To evaluate therapeutic potential, we performed blocking experiments with anti-CD44 monoclonal antibodies, which stopped tumorigenic proliferation

\begin{tabular}{|c|c|c|}
\hline \multicolumn{3}{|c|}{ Proteome Analyses } \\
\hline MS & 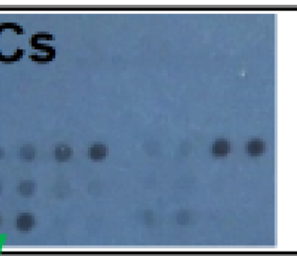 & $\begin{array}{l}\text { MCSCS } \\
y\end{array}$ \\
\hline ANGIOGENIC PROTEINS & Fold Changes & FUNCTION \\
\hline Angiogenin & 3.91 & initiates vascularization \\
\hline Angiopoietin-1 (Ang-1) & 1.44 & modulators of angiogenesis \\
\hline Angiopoietin-2 (Ang-2) & 1.42 & modulators of angiogenesis \\
\hline CD31 (PECAM-1) & 5.35 & promotes angiogenesis \\
\hline CD54 (ICAM-1) & 35.78 & promotes angiogenesis \\
\hline CD105 (Endoglin) & 4.45 & angiogenesis, and neovascularization \\
\hline CD106 (VCAM-1) & 11.51 & vascular cell adhesion molecule 1 (VCAM-1) \\
\hline EGF & 1.76 & associates with PDGF R beta and HGF R/c-MET \\
\hline FGF basic & 3.19 & growth factor that functions in angiogenesis \\
\hline FLT3LG & 1.86 & promotes tumor cell proliferation and invasion \\
\hline GM-CSF & 16.7 & promotes tumor cell proliferation and invasion \\
\hline HGF & 4.88 & $\begin{array}{l}\text { induces proliferation and migration of endothelial } \\
\text { cells }\end{array}$ \\
\hline LIF & 15.27 & promotes tumor cell proliferation and invasion \\
\hline PDGF-AA & 17.9 & promotes angiogenesis \\
\hline PDGF-AB/BB & 10.36 & promotes angiogenesis \\
\hline TFF3 & 6.68 & supports hypoxia-induced VEGF upregulation \\
\hline TNF-a & 22.5 & induces the activation of vascular endothelial cells \\
\hline VEGF & 3.34 & mediator of both angiogenesis and vasculogenesis \\
\hline $\begin{array}{l}\text { B } \\
\text { Fig. } 2 \text { Proteome analyses indicate the signific } \\
\text { developed for proteome analyses. The black } \\
\text { expression. The red arrowhead indicates prot } \\
\text { The green arrowhead indicates strong protei } \\
\text { manufacturer's specification. b Proteome com } \\
\text { cultures. Fold Changes represent MCSCs vers }\end{array}$ & $\begin{array}{l}\text { ant increase in angiogenic } \\
\text { rrow indicates the control } \\
\text { an expression at the same } \\
\text { expression at the same lo } \\
\text { parison (fold change) of ar } \\
\text { is MSCs }\end{array}$ & $\begin{array}{l}\text { protein release from MCSC cultures. a Image of partial blot films } \\
\text { dots from the manufacturer. The red arrow indicates no protein } \\
\text { location in the film. The green arrow indicates weak protein expression. } \\
\text { cation. Note: each antibody has two dot spots according to } \\
\text { agiogenic proteins between supernatants from P7 MSC and P7 MCSC }\end{array}$ \\
\hline
\end{tabular}


of P5 MCSCs, but not P7 MCSCs (Supplementary Figure 3). It is possible that the large amount of ICAM-1 (53-fold increase in P7 MCSCs versus P5 MCSCs, Supplementary Figure $3 \mathrm{E}$ ) compensated the loss of $\mathrm{CD} 44$ as previously reported in CD44 null mice [10].

In summary, we provide novel evidence of MCSCs as the cellular origin of angiogenesis and cytokine release in an AML patient's BM. More research with additional patients is needed to discover if this phenomenon is unique to this one AML patient or has broader ramifications for AML as a whole. Our data suggest that future therapeutic strategies should also be developed to target MCSCs and their cytokine release to achieve stringent complete remission and prevent AML progression and relapse. The existence of MCSCs might be applicable to other cancers as well.

\section{Supplementary information}

Supplementary information including Materials and Methods (Additional file 2) accompanies this paper at https://doi.org/10.1186/s13045-020-00899-X.

\section{Additional file 1: Supplementary Figures. Supplementary Figure}

1. There is no significant difference in differentiation capabilities of P4 MSC and P4 MCSCs during ex vivo cultures. A) MSCs Panel: FACS plot of CD34CD13+MSCs, which differentiated into bone (Alizalin staining), fat (phase bright), and cartilage (Alcian blue staining). B) MCSCS Panel: FACS plot of CD34-CD13+MCSCs, which differentiated into bone (Alizalin staining), fat (phase bright), and cartilage (Alcian blue staining). Supplementary Figure 2. Comparison of Cell Proliferation of MSCs and MCSCS. A) The P10 MCSCs were found to proliferate much faster than P10 MSCs. ${ }^{* *} P<0.01 ; B$ ) These rapidly proliferating MCSCs do not express cleaved Caspase3 (Cell Signaling Technology, Cat\#9664S) and continue to express strong CD44+. Supplementary Figure 3. Anti-CD44 monoclonal antibodies inhibited the cluster formation and proliferation of P5 MCSCs. A) Phase bright images of floating clusters from P5 MCSCs without treatment. B) Phase bright images of floating cells from P5 MCSCs with treatment of anti-CD44. C) Aggregate cluster count data from P5 MCSCs treated with anti-CD44 or without treatment. D) Aggregate cluster count data from P7 MCSCs treated with anti-CD44 or without treatment. Where applicable, data are means \pm SEM from each group and were analyzed by Student t-test. ${ }^{*} P<0.05 ; \mathrm{N}=3$. E) Proteome comparison (mean pixel density) of ICAM-1 between supernatants from P7 MCSC and P5 MCSC cultures. *P< 0.05

Additional file 2: Materials and Methods. Discovery of proangiogenic CD44+mesenchymal cancer stem cells in an acute myeloid leukemia patient's bone marrow

\section{Abbreviations}

AML: Acute myeloid leukemia; MSC: Mesenchymal stem cell; MCSCs: Mesenchymal cancer stem cells; BM: Bone marrow; HSCs: Hematopoietic stem cells; FACS: Flow cytometry; P: Passages

\section{Acknowledgements}

We thank Drs. Chung-Tsen Hsueh and Saied Mirshahidi for helpful comments.

\section{Authors' contributions}

Y.X. conceived the study and its design; J.X., H.C., and Y.X performed experiments and data analyses; Y.X. drafted the manuscript. All authors revised the manuscript. All authors read and approved the final manuscript.

\section{Funding}

This study is supported by the Loma Linda University Research Innovation Grant (to H.C.) and LLU GRASP (to Y.X.) These funding bodies had no role in the design or performance of the study nor in the writing or interpretation thereof.

\section{Availability of data and materials}

The datasets used and/or analyzed in the current study are available from the corresponding author.

\section{Ethics approval and consent to participate}

This research was approved by the Loma Linda University Institutional Review Board. The sample was provided upon written informed consent.

\section{Consent for publication}

Not applicable

\section{Competing interests}

The authors have filed for a patent $(62 / 947,118)$.

\section{Author details}

${ }^{1}$ Division of Hematology and Oncology, Loma Linda University Medical Center, Loma Linda, CA, USA. ${ }^{2}$ Regenerative Medicine, Department of Medicine, Loma Linda University, Loma Linda, CA, USA. ${ }^{3}$ Division of Anatomy, Department of Basic Sciences, Loma Linda University, Loma Linda, CA, USA ${ }^{4}$ Gehr Family Center for Leukemia Research, Hematology Malignancies and Stem Cell Transplantation Institute, City of Hope Medical Center, Duarte, CA, USA.

Received: 16 March 2020 Accepted: 15 May 2020

Published online: 03 June 2020

\section{References}

1. Almeida AM, Ramos F. Acute myeloid leukemia in the older adults. Leuk Res Rep. 2016;6:1-7.

2. Medeiros BC, et al. Big data analysis of treatment patterns and outcomes among elderly acute myeloid leukemia patients in the United States. Ann Hematol. 2015;94(7):1127-38.

3. Bruserud $\mathrm{O}$, et al. Subclassification of patients with acute myelogenous leukemia based on chemokine responsiveness and constitutive chemokine release by their leukemic cells. Haematologica. 2007;92(3):332-41.

4. Kittang $\mathrm{AO}$, et al. The chemokine network in acute myelogenous leukemia: molecular mechanisms involved in leukemogenesis and therapeutic implications. Curr Top Microbiol Immunol. 2010;341:149-72.

5. Kumar B, et al. Acute myeloid leukemia transforms the bone marrow niche into a leukemia-permissive microenvironment through exosome secretion. Leukemia. 2018;32(3):575-87.

6. Ganzel C, et al. Very poor long-term survival in past and more recent studies for relapsed AML patients: The ECOG-ACRIN experience. Am J Hematol. 2018;93:1074-81.

7. Kode $\mathrm{A}$, et al. Leukaemogenesis induced by an activating beta-catenin mutation in osteoblasts. Nature. 2014;506(7487):240-4.

8. Raaijmakers $\mathrm{MH}$, et al. Bone progenitor dysfunction induces myelodysplasia and secondary leukaemia. Nature. 2010;464(7290):852-7.

9. Cogle $\mathrm{CR}$, et al. Functional integration of acute myeloid leukemia into the vascular niche. Leukemia. 2014;28(10):1978-87.

10. Olaku V, et al. c-Met recruits ICAM-1 as a coreceptor to compensate for the loss of CD44 in Cd44 null mice. Mol Biol Cell. 2011;22(15):2777-86.

\section{Publisher's Note}

Springer Nature remains neutral with regard to jurisdictional claims in published maps and institutional affiliations. 